59. Tagung

Jahrestagung vom 18.-20. September 1986

in Aachen, gemeinsam mit den

Neurologischen Gesellschaften der Beneluxländer 


\section{Verhandlungen der \\ Deutschen Gesellschaft für Neurologie \\ 4}

Herausgegeben von Neuroimmunologie

K. Poeck, W. Hacke Spinale Krankheiten

und R. Schneider Neuropsychologie

Metabolische Enzephalopathien

Neurologische Notfälle

Interventionelle Neuroradiologie

Springer-Verlag

Berlin Heidelberg New York

London Paris Tokyo 


\section{Inhaltsverzeichnis}

Verzeichnis der Autoren und Vortragenden

I. Neuroimmunologie

Autoimmunmechanismen bei neuromuskulären Erkrankungen

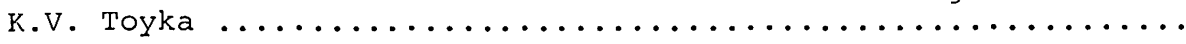

Cellular and Humoral Components of the Cerebrospinal Fluid

in Multiple Sclerosis

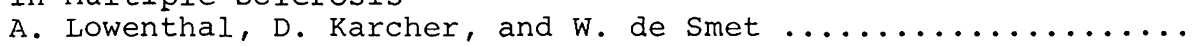

Das Konzept zentralnervöser Schranken in der Neuroimmunologie

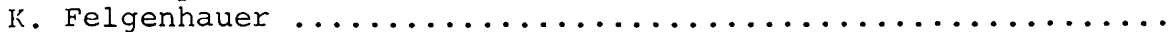

Tiermodelle des Guillain-Barré-Syndroms

H. Wiethölter, M. Schabet und P.-J. Hülser $\ldots \ldots \ldots \ldots \ldots \ldots \ldots$

Cyclosporin A in der Behandlung von multipler Sklerose und Myasthenie

L. Kappos

Experimental Allergic Encephalomyelitis and Immunosuppression

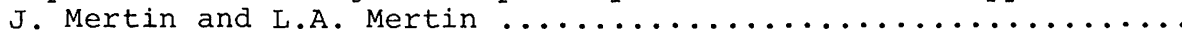

Progressive Borrelia Encephalomyelitis - A Chronic Neurological Manifestation of Erythema Migrans Borreliosis

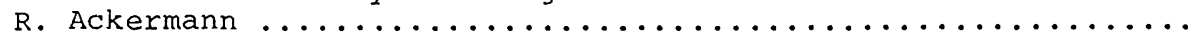

Beteiligung des Nervensystems bei AIDS

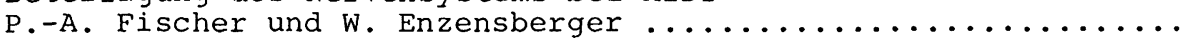

Neuropathological Findings in AIDS Patients

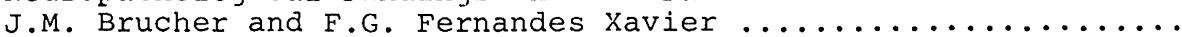

Indications for Plasma Exchange in Neurology

H.J.G.H. Oosterhuis and A.E.J. de Jager ...............

Immunotherapy in Multiple Sclerosis: Fact or Pancy?

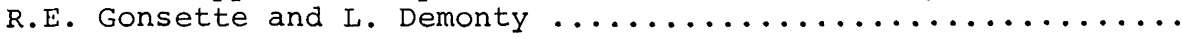

Einsatz von bildgebenden Verfahren (Computertomographie/CT und Kernspintomographie INMR) bei entzündlichen Muskelerkrankungen (Poly/Dermatomyositis - PM/DM: Möglichkeiten in Diagnostik und Therapiekontrolle

B. Schalke, W.A. Kaiser, G. Schindler und R. Rohkamm ........ 
Signifikanz der Serum- und Liquorbefunde bei primär entzündlichen INeuropathien

H. Krüger, O.G. Segurado, R. Martin und H.G. Mertens ........

Changes of the Blood-Nerve Barrier During Nerve Regeneration in the Mouse

R.J. Seitz, K. Reiners, K. Heininger, H.P. Hartung,

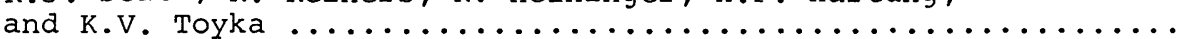

Regeneration des peripheren Nerven bei experimentellen Immunneuropathien

K. Reiners, G. Stoll, G. Schwendemann, K. Heininger und

K.V. Toyka ..................................

Histologische Befunde an der skelettmuskulatur bei

Immunvaskulitiden

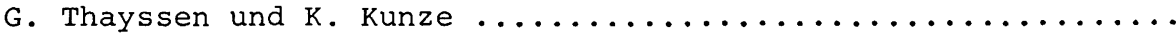

Periodic Fluctuations in Cerebrospinal Fluid Mononuclear Cells C.H. Polman, C.J.A. de Groot, J.C. Koetsier, T. Sminia, and

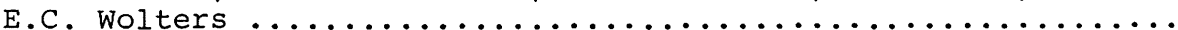

Plasmaaustauschbehandlung bei Poly- und Dermatomyositis (PM/DM)

R. Rohkamm, P. Reuther und B. Schalke .................

Akut- und Lanzeitverläufe bei Myasthenia gravis: Konsequenzen fir die immunsuppressive Therapie

T. Emskötter, L. Lachenmayer und K. Kunze ...............

Selective Adsorption Therapy in Myasthenia Gravis

K. Heininger, H.-P. Hartung, A. Gaczkowski, H. Borberg,

B. Grabensee, and K.V. Toyka ......................

T Lymphocytes Specific for Acetylcholine Receptor: Isolation from Thymuses of Myasthenia Gravis Patients

A. Melms, B. Endler, B. Schalke, and H. ritekerle ...........

Lymphocyte Subpopulations in Cerebrospinal Fluid: Multiple Sclerosis and Other Neurological Disease Categories

C.H. Polman, C.J.A. de Groot, J.C. Koetsier, and T. Sminia ...

Lymphozyten-Subpopulationen im Liquor- methodische Probleme

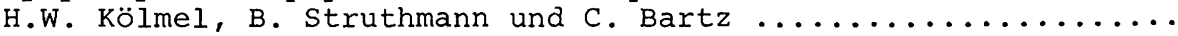

Deutsche Multicenterstudie Cyclosporin A versus Azathioprin Methodische Aspekte

S. Poser, L. Kappos, U. Patzold und D. Dommasch ............

Deutsche Multicenterstudie Cyclosporin A versus Azathioprin: Nebenwirkungen der Behandlung - Kosten, Nutzen, Analyse S. Poser, U. Patzold, L. Kappos, D. Dommasch, P. Krauseneck, J.P. Malin, K. Wonigeit, J. Haas, W. Fierz und U. Wurster ....

Cyclosporin A bei multipler Sklerose - Ergebnisse der Deutschen Multicenterstudie

U. Patzolả, D. Dommasch, J. Maas, L. Kappos und S. Poser ..... 
II. Spinale Krankheiten

Traumatische Querschnittlähmung - konservative und chirurgische Behandlung in der Frühphase

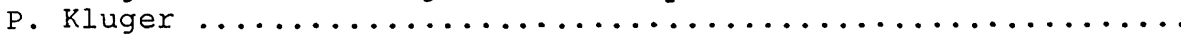

Neuroprostheses in Transvers Lesions of the Cervical Cord

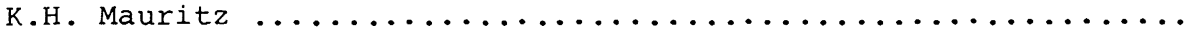

Pharmakotherapie bei Blasenstörungen

J. Hannappel .................................

Chemonucleolysis

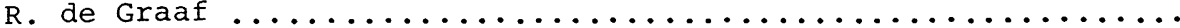

Die symptomatisch atlanto-axiale Instabilität - Indikation,

Zeitpunkt und Fortschritte der operativen Versorgung

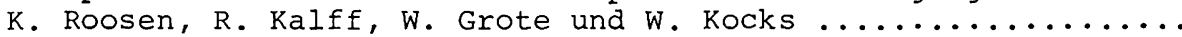

Operative Stabilisierungsverfahren bei Verletzungen der Halswirbelsäule

R. Kalff, W. Kocks, K. Roosen, W. Grote und U. Schüwer .......

Ergänzung der Mikrochirurgie durch Überwachung somatosensorischer Bahnen in der spinalen Tumorchirurgie

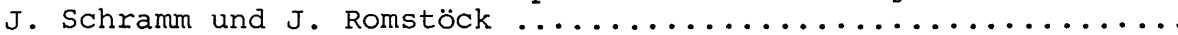

Neurophysiologische Verlaufsdokumentation sensomotorischer

Funktionen nach operativen Eingriffen am Rückenmark

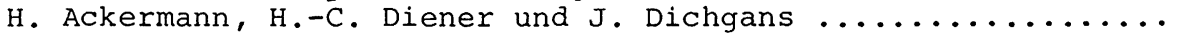

Postpolio Syndrome: New Name or New Diagnosis?

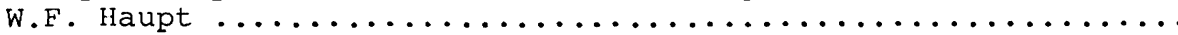

Die operative Therapie der Syringo- und Hydromyelie nach

Einführung der NMR

N. Hüwel

Spinale Tumoren im Kindesalter - kombiniertes neuro-

chirurgisches und radiologisches Therapiekonzept

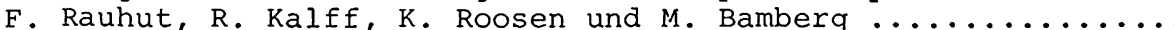

\section{Neuropsychologie}

Quantitative dreidimensionale Bilddarstellung in der Neuropsychologie

G. Pawlik, W.-D. Heiss, I. Hebold, C. Beil, K. Herholz

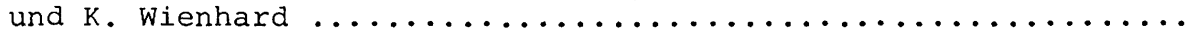

Regional Cerebral Blood Flow Studies During Functional Tests in Aphasic Stroke Patients

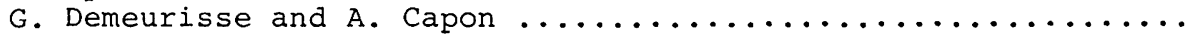

Möglichkeiten und Grenzen des Gesichtsfeldtrainings bei Patienten mit postgenikulär bedingten Gesichtsfeldausfällen

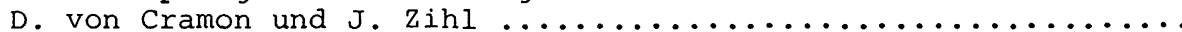

Neurolinguistic Approach of Dementia

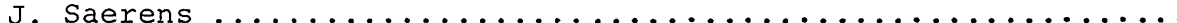


Counting by Aphasics

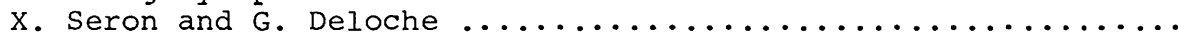

Blickbewegungen während linguistischer Verarbeitung bei

Aphasiepatienten

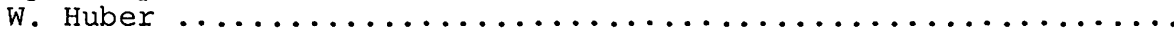

Lateralisiertes Training nichtverbaler kognitiver Fähigkeiten bei aphasischen patienten

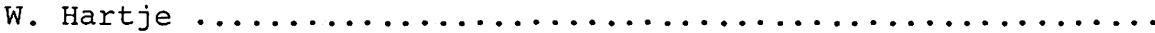

Transient Global Amnesia

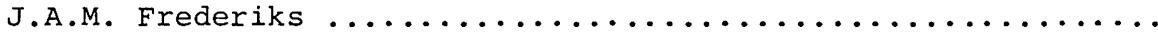

Auftretensbedingungen sprachlicher Automatismen

J.C. Haas, P. Kronthal, G. Blanken und C.-W. Wallesch ........

Pure Anarthria Without Simultaneous Buccofacial Apraxia in a Luxemburgish Patient

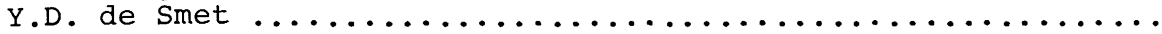

Kognitive Potentiale (P3, P300): Methode und Probleme der

klinischen Anwendung

W. Emser, G. Heinz, K. Schimrigk, H. Giener und M. Weiland ....

Ereignisbezogene Potentiale (P300) in der Diagnostik

dementiver Erkrankungen

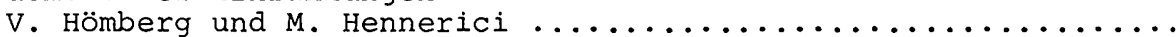

Untersuchungen des konditionierten Lernverhaltens von Kranken mit degenerativen zerebellären Erkrankungen unter Berücksichtigung der $\mathrm{CNV}$

K. WeiBenborn, M. Scholz, H.-J. Heinze, H. Feistner und

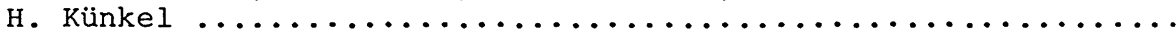

Der "Akense Afasietest" - eine niederländische Fassung des AAT (Aachener Aphasietest)

R. de Bleser, P. Graetz, P. Hagoort, C. Heeschen, A. Keyser,

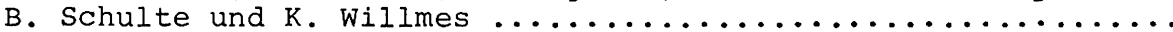

Der "Akense Afasietest" - Erfahrungen und Probleme bei der

Validierung

C. Heeschen, R. de Bleser, P. Graetz, P. Hagoort, A. Keyser,

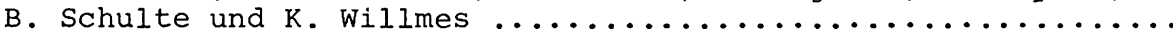

Neuropsychological Impairment Associated with Thalamic Ischemic Lesions

P. Herregodts, V. Morez, A. Michotte, C. Solheid, and

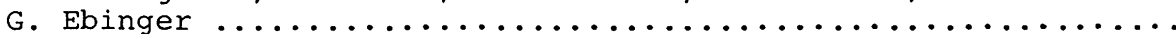

Spontanverlauf vaskulärer, retrogenikulärer Gesichtsfeldstörungen

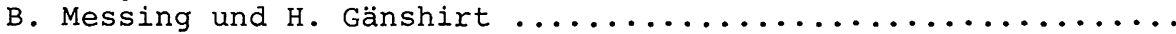

Der EinfluB von lateralisierten Warnreizen auf eine visuelle Wahlreaktionsaufgabe

$\mathrm{W}$. Sturm, J. Reul und $\mathrm{K}$. Willmes $\ldots \ldots \ldots \ldots \ldots \ldots \ldots \ldots \ldots \ldots$

Right Frontal Lobe Dominance in Spatial Visuomotor Learning:

Evidence from Electrophysiological Experiments

W. Lang, M. Lang, A.W. Kornhuber, and H.H. Kornhuber ........ 
Zur struktur des hirnorganischen Psychosyndroms

$\mathrm{J}$. Hunger, C. Mohs, B. Leplow und J. Kleim ...............

Cheiro-oral Syndrome: The Myth of Its Localizing Value

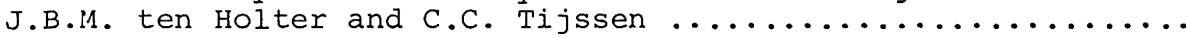

IV. Metabolische Enzephalopathien

Metabolische Enzephalopathien

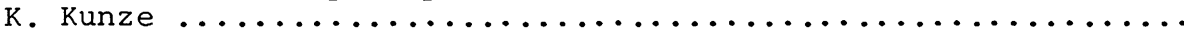

Encephalomyopathies with Abnormal Mitochondria

G.F. Walter, J.J. Martin, and J.M. Brucher ...............

Clinical and Metabolic Aspects of Leigh Syndrome

P.M.M. van Erven, F.J.M. Gabreëls, W. Ruitenbeek, R.A. Wevers,

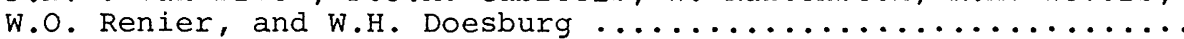

Enzephalopathien bei Nierenkrankheiten

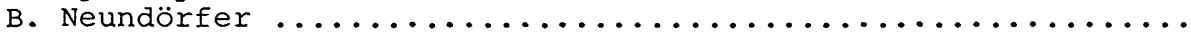

Lafora Disease: Diagnosis and Carrier Detection

H.L.S.M. Busard, W.O. Renier, F.J.M. Gabreëls, and

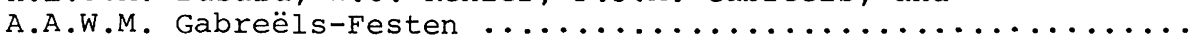

Blood and CSF Concentration of Fuel Related Components

in Children After Prolonged Fasting

K.J.B. Lamers, W.H. Doesburg, F.J.M. Gabreëls, R.A. Wevers,

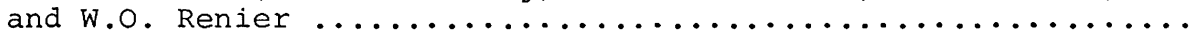

Spinozerebelläre Atrophien - Diagnose und Therapie

H. - G. Mertens und $\mathrm{W}$. Kohlhepp .......................

Bilanzierung der Ein- und Ausfuhr von Kupfer mit Chelatbildnern und Zinksulfat bei Morbus Wilson

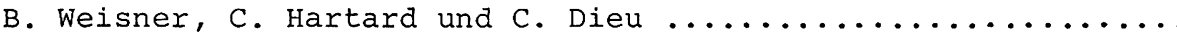

Therapeutic Problems in Patients Suffering from Aluminium

Encephalopathy

C.E. Elger, O. Knoll, A.C. Ludolph, and H. Raidt ...........

Dihydrothymine Dehydrogenase Deficiency: A Cause of Cerebral Dysfunction?

J.P. Braakhekke, W.O. Renier, F.J.M. Gabreëls, R.A. de Abreu,

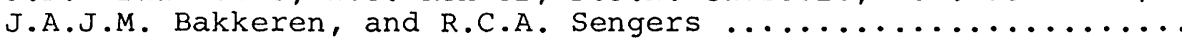

EinfluB von 1-Methyl-4-phenyl-1,2,3,6-tetrahydrophyridin (MPTP), Budipin und Biperiden auf den Substanz-P-Gehalt des Nucleus caudatus

K. Henning, P. Wallasch, H. Ruß und H. Przuntek ...........

Enzymatic Analyses of Beta-Oxidation in Human Fibroblasts from Patients with Reye's Syndrome

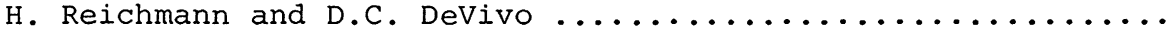

Diagnostische Hinweise bei neurometabolischen Erkrankungen

im Kindesalter

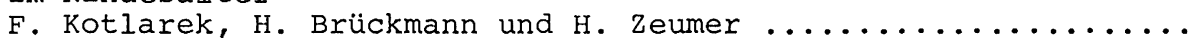


Ataxia Telangiectasia: A Disease Exclusively of Childhood?

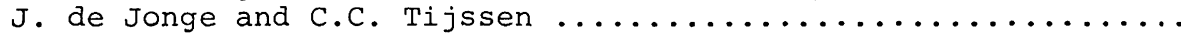

Porphyrie - Enzephalopathie

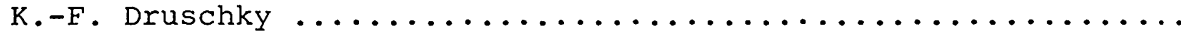

Alcoholic Brain Damage: Possible Cause of Epilepsy

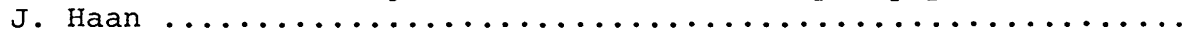

Untersuchungen möglicher Ursachen der Hirnvolumenänderung abstinenter Alkoholiker

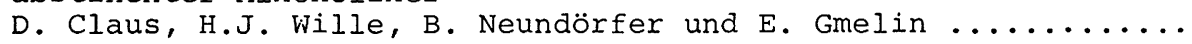

Blink Reflexes in Patients with Metabolic Encephalopathy

L.J. Dénes, M.D. Tavy, H.E. Humphry, M.D. Morré,

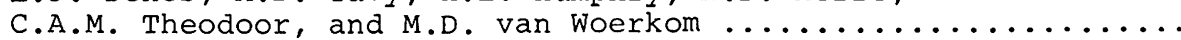

Milde Verlaufsformen der zentralen pontinen Myelinolyse

H.W. Pfister, K.M., Einhäupl, T. Brandt und M. Naegele .......

CT-Verlauf und G-Protein - Untersuchungen bei symmetrischen

zerebralen Stammganglienverkalkungen (Fahr-Syndrom)

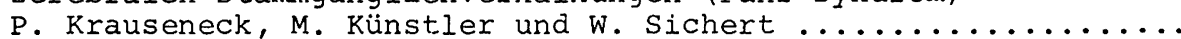

Metabolisch-toxische Enzephalopathien-ihre Begleitzeichen

im EEG

J. Kugler

V. Neurologische Notfälle

The Treatment of Malignant Status Epilepticus

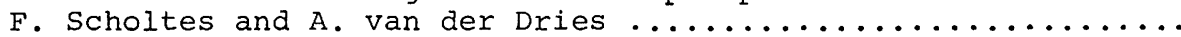

Botilismus und Tetanus

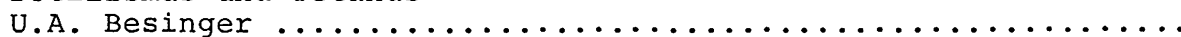

Die aneurysmatische Subarachnoidalblutung: Diagnose und konservative Therapie

A. Ferbert

Akute Virusenzephalitis und Enzephalopathie (ohne Herpesenzephalitis)

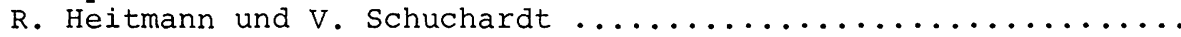

Anoxisches Koma: Pathomechanismen, klinische Syndrome,

Therapieüberlegungen

R.W.C. Janzen

Treatment of Severe Closed Head Injury

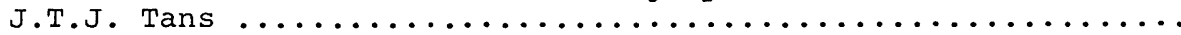

The Pay-off of Brain Protection

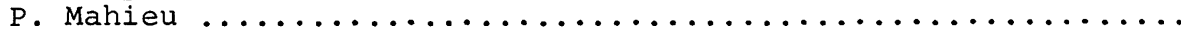

VI. Interventionelle Neuroradiologie

Interventional Neuroradiology of Ear, Nose and Throat Conditions in the Neighbourhood of Neuronal Structures

P. Mathurin 
Elektrophysiologisches Monitoring bei neuroradiologischen Eingriffen

H. Buchner, H. Brückmann, A. Ferbert, H. Zeumer und W. Hacke ..

VII. Freie Themen

Postersitzung - Multiple Sklerose

Akute experimentell-allergische Enzephalomyelitis als Modell für Einflüsse entzündlicher zentraler Paresen auf den monosynaptischen spinalen Reflexbogen

J. Rimpel, K. Buschhorn, B. Emmerich und H.J. Lehmann ........

Determination of the Antibody Activity of CSF Oligoclonal

Bands by Immunoblotting

K. Bukasa, J. Limet, E.C. Laterre, and C.J.M. Sindic ........

Thrombozytenfunktion und multiple Sklerose

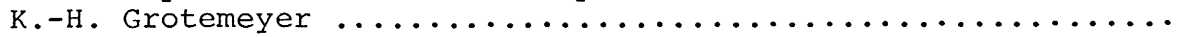

Inthratekale vs. orale Kortikosteroidtherapie von spinalen Symptomen bei multipler Sklerose (eine kontrollierte Doppelblindstudie)

E. Rohrbach, L. Kappos, D. Städt, A. Hennes, D. Kaiser,

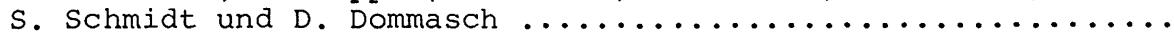

Morbus Schilder - eine Variante der MS? - Unterschiede und Gemeinsamkeiten (kasuistischer Beitrag)

E. Rohrbach, L. Kappos, R. Meyermann und M. Ratzka ..........

Azetylcholin-Rezeptoren-Antikörper bei amyotropher Lateralsklerose (ALS)

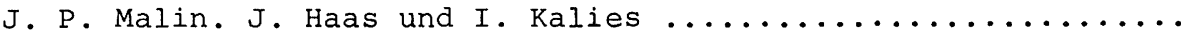

Kopfschmerzen - Initialsymptom der Encephalomyelitis

disseminata?

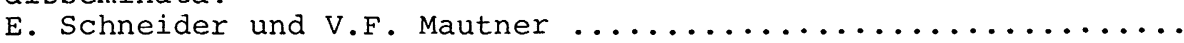

Postersitzung - Myasthenie

Phencyclidin hemmt die Transmitterfreisetzung an der neuromuskulären Synapse der Heuschrecke

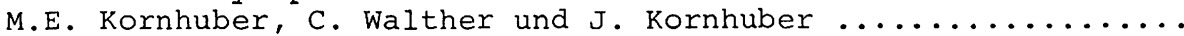

Erste Ergebnisse einer kontrollierten doppelblind durchgeführten Therapiestudie: Cyclosporin (Ci-A) vs. Azathioprin

( Aza) bei Myasthenia gravis

B. Schalke, L. Kappos, E. Rohrbach, D. Dommasch,

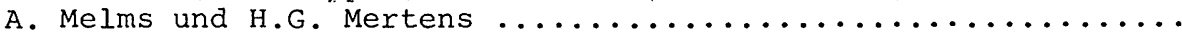

Immunhistochemische Befunde im tumorfreien Thymus bei

Myasthenia gravis

T. Kirchner, H.K. Müller-Hermelink und B. Schalke ..........

Antibodies to Acetylcholine Receptors (Ab) and Clinical

Condition During Various Treatment Modalities in Myasthenia

Gravis: A Follow-up Study

H.J.G.H. Oosterhuis, P.C. Limburg, E. Hummel-Tappel, G. Horst,

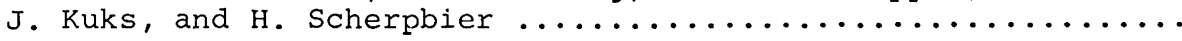


High Dose 7S-Immunoglobulin Treatment in Myasthenia Gravis S. Knorr-Held, M. Wick, H. Kissel, A. Fateh-Moghadam,

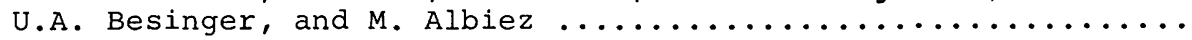

Feinspezifität von Autoantikörpern bei Myasthenia-gravisPatienten

B. Kohleisen, G. Leipold, W. Stöcker, K.-F. Druschky,

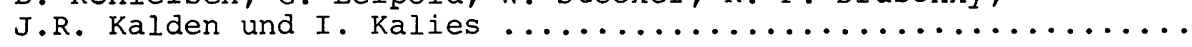

Verminderte Con-A-Reaktivität der Suppressorzellpopulation

bei Myasthenia-gravis-Patienten

H. Kachelries, I. Kalies, P. Rohwer, J.R. Kalden und

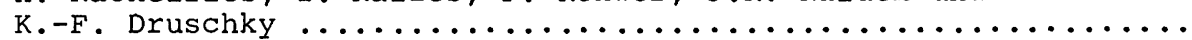

Effect of Thymectomy on Myasthenia Gravis and Autoimmune

Thrombocytopenic Purpura in a 13-Year-Old Girl

P.H.P. Jansen, W.O. Renier, G. de Vaan, P. Reekers, and

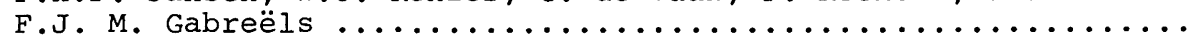

Postersitzung - Liquor

Angiotensin-Converting-Enzyme (ACE) im Liquor cerebrospinalis: erhöhte Werte der spezifischen Aktivität bei akuter Sarkoidose und Chorea Huntington

W. Kuhn, H. Schweisfurth, S. Schiöberg-Schiegnitz, W. Bobbert,

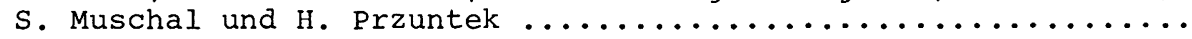

Nachweis und Identifizierung Borrelia-burgdorferi-spezifischer, oligoklonaler Immunglobuline ( $I g G)$ im unkonzentrierten Liquor

mit der Immunoblottechnik

R. Martin, U. Martens, H. Krüger, V. Sticht-Groh und

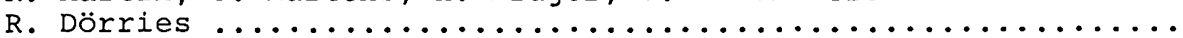

Intrathekale Produktion spezifischer Antikörper gegen Borrelia burgdorferi bei Patienten mit Bannwarth-Syndrom

B. Wilske, G. Schierz, V. Preac-Mursic, L. Bader,

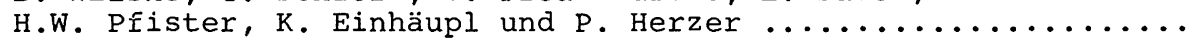

Ergebnisse liquorzytologischer Untersuchungen mit monoklonalen Antikörpern

E. Stark

Immunzytochemische Untersuchung von T-Zell-Subsets in Liquor und Blut bei entzündlichen Erkrankungen des Nervensystems S. Jürgens, T. Weber, P. Rieckmann, H. Prange und

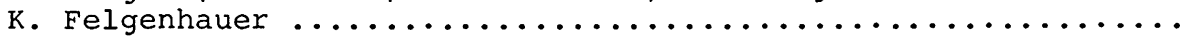

Central Nervous System Involvement in a Symptomatic Male Syphilitic Human-Immunodeficiency-Virus Carriers

E.C. Wolters, J. Goudsmit, R.V.W. van Eijk, and

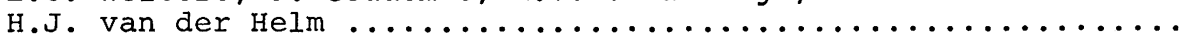

Somatostatin im Liquor von Patienten mit Zosterneuralgie

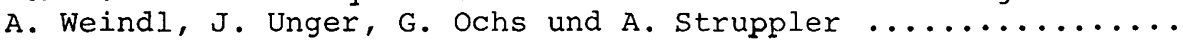

Aprotinin Versus Tranexamic Acid in Antifibrinolytic Treatment of Patients with Subarachnoid Hemorrhage

F.J. von Baumgarten, G. Burkard, D. Englert, H.G. Mertens,

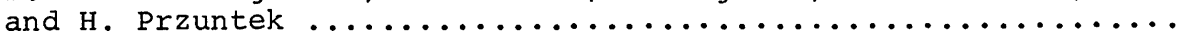


Einfluß der Blutkontamination im "klaren" Liquor cerebrospinalis auf die Aminosäurekonzentration

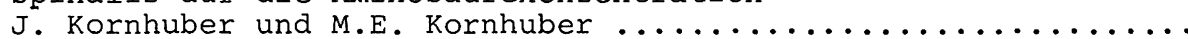

HTLV-III-Infektion - Zell- und Eiweißbefunde im Liquor

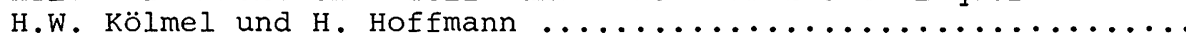

Postersitzung - Multiple Sklerose, Liquor

Immunglobulinproduzierende Zellen im Liquor von Patienten mit entzündlichen Erkrankungen des Zentralnervenșystems (ZNS)

P. Rieckmann, S. Jürgens, T. Weber, H. Prange und

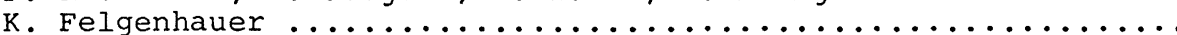

Appearance and Distribution of Macrophages in the Central Nervous System of Lewis Rats with Acute Experimental Allergic Encephalomyelitis: An Immunohistochemical Study

C.H. Polman, C.D. Dijkstra, C.J.A. de Groot, J.C. Koetsier,

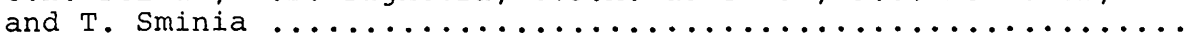

Experimental Measles Enceophalomyelitis in the Rat: Generation of Measles Virus (MV) and T-Lymphocyte Cell Lines Specific for Myelin Basic Protein (MBP)

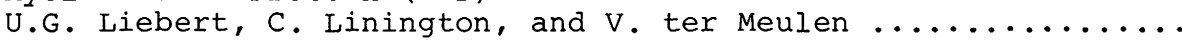

Multiple Sklerose (MS) und humane lymphotrope Retroviren: negative serologische Befunde bei 135 MS-Patienten

J. Schneider, B. Kitze, V. ter Meulen, G. Hunsmann,

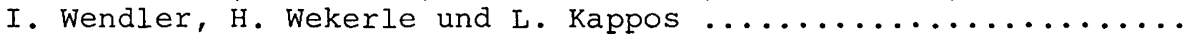

Vergleichende Untersuchungen zur Bestimmung der Blut-Liquor-

Schrankenstörung und der intrathekalen IgG-Synthese

S. Horn, H. Krüger, K. W. Pflughaupt und H.G. Mertens ........

Intraocular IgG Synthesis in Multiple Sclerosis Patients

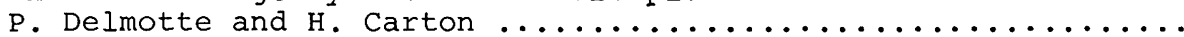

Polyneuritis und multiple Sklerose

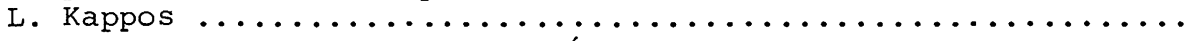

Immunsuppressive Therapie von an multipler Sklerose (MS) und Myasthenie (MG) erkrankten Patienten mit Azathioprin: Nebenwirkungen im Langzeitverlauf

L. Kappos, U. Stolle, U. Wilhelm, E. Rohrbach und

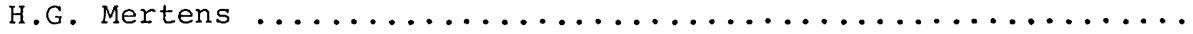

A Fetuin-like Antigen in MS Sera: An Immunosuppressive Factor? C.J.M. Sindic, M.P. Chalon, O.C. Fagnart, and E.C. Laterre ....

Postersitzung - Liquor, peripherer Nerv

Therapieversuch der amyotrophischen Lateralsklerose mit dem Kalziumantagonisten Flunarizin

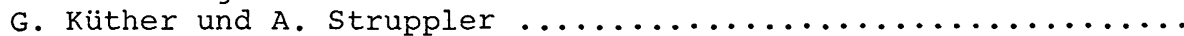


XVI

Immunhistologische Darstellung des Gangliosids $G_{M} 1$ im

Skelettmuskel bei Neuropathien und Myopathien

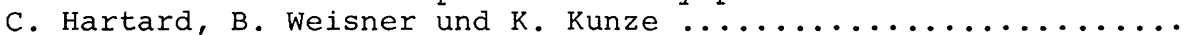

Agenesis of the Corpus Callosum in a Case of Infantile Spinal Muscular Atrophy

F. Scholtes, F.M. Gabreëls, H. Jasner, and r. Renier ........

Die relative Refraktärperiode des Nervus suralis in der

klinischen Diagnostik polyneuropathischer Syndrome

I.W. Husstedt, K.H. Grotemeyer und H.P. Schlake ............

Role of Macrophages in Peripheral Nerve Regeneration

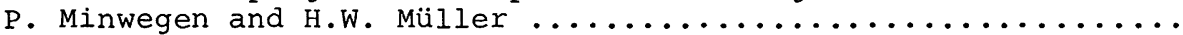

Antivirale Antikörper und Verlaufsdynamik bei der multiplen Sklerose

H. Karbe, H.-J. Schädlich, M. Nekic und K. Felgenhauer .......

Freie leichte Ketten im Liquor bei multipler Sklerose

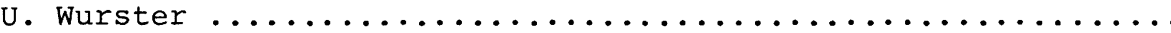

Der GABA-Gehalt im Liquor bei Querschnittssyndrom, Chorea

Huntington und anderen neurologischen Erkrankungen

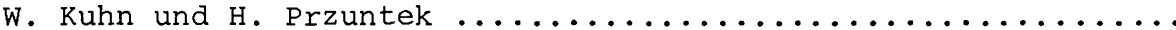

Quantitativer Nachweis von Substanz $\mathrm{P}$ aus menschlichem Liquor cerebrospinalis

T.-M. Wallasch, K. Henning, H. Eckhardt-Wallasch, W. Kuhn

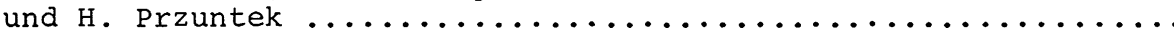

Signifikanz der Serum- und Liquorbefunde bei primär entzündlichen Neuropathien

H. Krüger, O.G. Segurado, R. Martin und H.G. Mertens .........

Postersitzung - Peripherer Nerv, Polyneuritis

Clinical Observations and Investigations of Pathogenicity

in the POEMS Syndrome

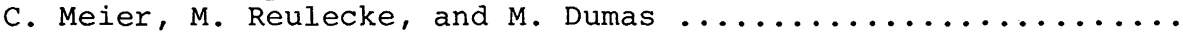

Karnitinkonzentrationen in Serum, Urin und Muskel bei neurogenen und myogenene Muskelatrophien

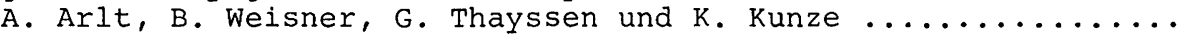

Polyneuropathie bei HTLV-3-Infektion

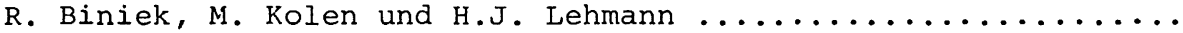

Liquorzytologie bei Guillain-Barré-Syndrom

A. Engelhardt, E. Herb, B. Neundörfer und $\mathrm{K}$. Warecka .........

Suppression and Treatment of EAN by Novel Antiinflammatory

Agents Acting on Macrophages

H.P. Hartung, B. Schäfer, K. Heininger, G. Stoll, and

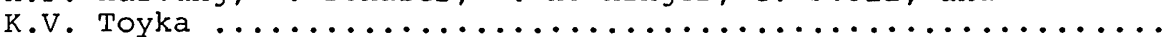

Polymyositis (PM) : Mehrfachmarkierung und Phänotypisierung mononukleärer Zellen (MNZ) in Infiltration und im Blut: Analyse im Fluoreszenz- und Laser-Scan-Mikroskop (LSM)

W. Schubert 
Polyneuropathien durch Blei, Thallium und Gold

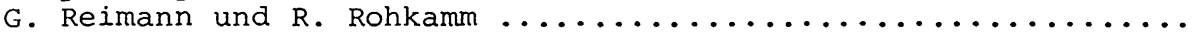

Akute Femoralisparese bei spontanem retroperitonealen Hämatom unter Antikoagulanzientherapie - eine dringende chirurgische Indikation

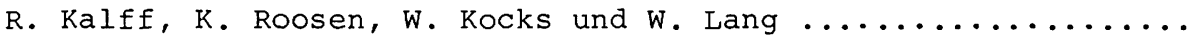

Postersitzung - Intensivmedizin I

Evozierte Potentiale bei Basilaristhrombose

A. Ferbert, H. Buchner, W. Hacke, H. Bruckmann, R. Drummen

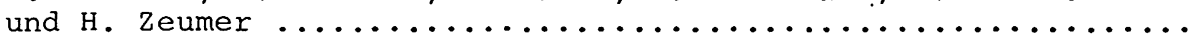

Zur Einteilung der BewuBtseinslagen

D. Moskopp, F. Ries, H.F. Durwen und D.B. Linke ............

Die prognostische Bedeutung vertikaler Blickdeviationen im Koma

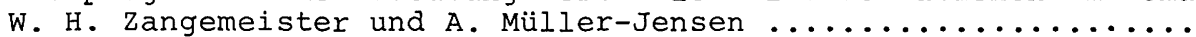

Die Behandlung des malignen neuroleptischen Syndroms mit Lisurid

H. Przuntek, E. Epping, H. Beckmann, I. Suchy, S. Bittkau

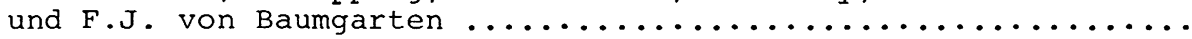

Mortalität ischämischer Hirninfarkte

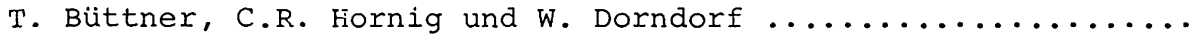

Vergleichende Untersuchung von Aprotinin und Tranexamsäure zur Prophylaxe der rezidivierenden Subarachnoidalblutung und Untersuchung zu Risikofaktoren für die spasmusbereitschaft bei Subarachnoidalblutung

G. Burkard, F.J. von Baumgarten, H. Englert und H. Przuntek...

Early Diagnosis and Elective Neurosurgical Treatment of Familial Intracranial Aneurysms: Report of Two Families J.W.M. ter Berg, J. Willemse, J. Bijlsma, J.W. Ludwig, and C.A.F. Tulleken ............................

Klinik und Prognose der spontanen Ventrikelblutung

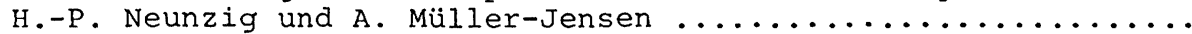

Akute subkortikale Demyelinisierung bei benigner Gammopathie A. Thie, R. Laas, J. Löhler, D. Müller, K. Spitzer und K. Kunze

Postersitzung - Intensivmedizin II

Therapie chronisch-hypoglykorrhagischer Meningitiden

F. J. von Baumgarten, S. Bittkau, A. Burkhard, K. Höhnke,

G. Krichenbauer, H.G. Mertens und H. Przuntek ..............

Listerien-Rhombenzephalitis: NMR-Befund und Elektrophysiologie

G. Krämer, R. Besser, M. Just, S. Schuster, L. Weilemann,

F. Thömke, M. Hartmann und H.C. Hopf ...................

Familial Meningococcal Disease H.M.C. Steenstra, E.J. Kuijper, C.C. Tijssen, A. Jansz, H.C. Zanen, and A.G. Sjöholm ..................... 
Kryptokokkus-Meningoenzephalitis bei AIDS

W. Enzensberger, P.-A. Fischer, H. Gräfin Vitzthum, W. Schlote,

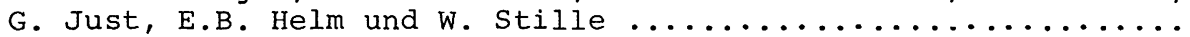

Neurologische Erkrankungen bei zeckenvermittelter Borreliose

W. Kohlhepp, H.G. Mertens, P. Oschmann und E. Rohrbach .......

Herpes-simplex-Enzephalitis - Therapie und Verlauf

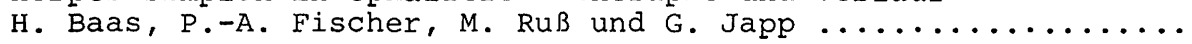

Anterior Fontanelle Pressure Monitoring Using the Rotterdam

Teletransducer and the Pressure Depth Curve

W.C.G. Plandsoen, D.A. de Jong, J.H.M. van Eijndhoven, and

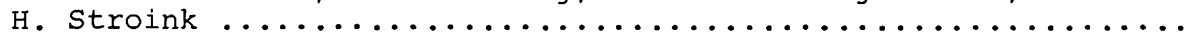

Stellenwert der transkraniellen Doppler-Sonographie in der Hirntoddiagnostik

F. Ries und D. Moskopp

Foudroyante oxalatenzephalitis nach hyperkalorischer

parenteraler Ernährung

C.R. Hornig, T. Büttner, J. Reiner, W. Schachenmayr und

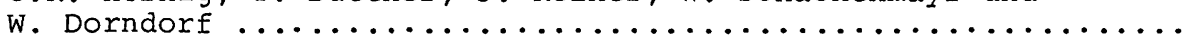

Postersitzung - Schlaganfall

"Trans-Scan" (3-D) transkranielle Doppler-Sonographie

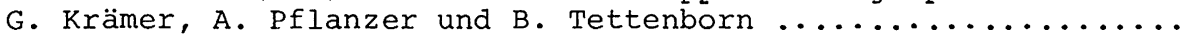

Zur Bedeutung des Anti-Elastin-Antikörpertiters bei Schlaganfallpatienten

$\mathrm{H}$. Henningsen, $\mathrm{C}$. Kessler und $\mathrm{R}$. Reuther $\ldots \ldots \ldots \ldots \ldots \ldots$

Effect of Stable Xenon on Regional Cerebral Blood Flow in Normal Volunteers

A. Hartmann, F. Schuier, Y. Tsuda, H. Wassmann, C. Dettmers,

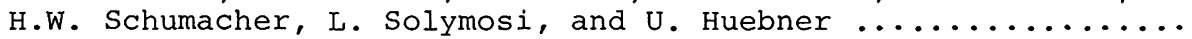

Alteration of Cerebral Blood Flow and Hemorheologic Parameters by Infusion of Hyperosmolar Solutions in Patients with Brain Edema

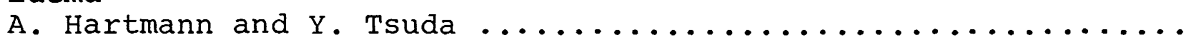

Indium-111-Plättchenszintigraphie bei Schlaganfallpatienten während der Infusion eines PGI 2 -Analogons

C. Kessler, H. Eenningsen, R. Reuther, H. Bihl und T. Steinmetz

Regional Cerebral Blood Flow Studies with 123I-Amphetamine

(IMP) SPECT and $133 \mathrm{X}$-Inhalation in Human Cerebrovascular

Disorders as Compared with Findings in Normal Baboons

Y. Tsuda, A. Hartmann, H.J. Biersack, E. Valerius, W. Baulig,

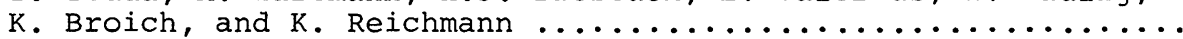

SEP Prognosis in Acute Stroke

J.W. Vredeveld ...............................

Neurologische Manifestation der Vaskulitiden

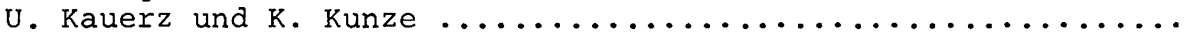

Die Aachener Feldstudie. Eine dopplersonographische Reihenuntersuchung an den extrakraniellen Hirnarterien

G. Leonhardt, R. Sonnenberg, K. Willmes und E.B. Ringelstein .. 
Electrophysiological and Sonographical Evaluation of Basilar Artery Blood Flow in Patients with Subclavian Steal Mechanism M. Busker, H. Buchner, E.B. Ringelstein, and W. Hacke ........

Postersitzung - Neuroradiologie

Diagnostic and Therapeutic Options in Stenosis of the Cervical Spinal Canal

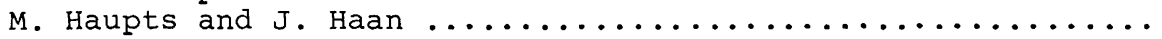

Computed Tomography (CT)-Controlled History of Conservatively Treated Acute Lumbar Disc Lesions

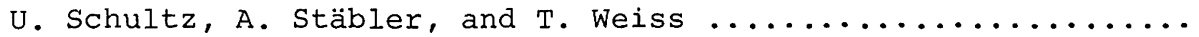

Unterschiedliche Topologie und Entwicklungsdynmakik bei Herpessimplex-Enzephalitis im Säuglings-, Kindes- und Erwachsenenalter

H. Brückmann, W. Hacke, H. Zeumer und F. Kotlarek ...........

Neurological Aspects of Selective Embolization of Cerebral

Arteriovenous Malformations

A.A.W. Op de Coul, J.P. ter Bruggen, J.B.M. ten Holter,

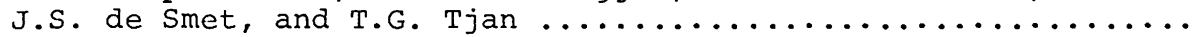

An Unusual Congenital Arteriovenous Fistula of the Vertebral

Artery and Its Embolization Using a Detachable Balloon Catheter

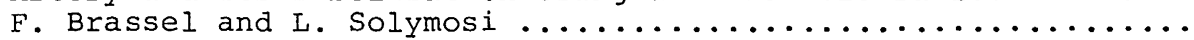

Computer-Assisted Anatomical Localization of Cortical Areas

D.G. von Keyserlingk, K. Poeck, and K. Niemann .............

Differentialdiagnostische Wertigkeit uni- und multilokulärer signalintenser Zonen in der Kernspintomographie

H. Goossens-Merkt, H. Krömer, H. Traupe und $K$. Kunze ........

Postersitzung - Epilepsie

Immunglobuline unter antiepileptischer Langzeitmedikation

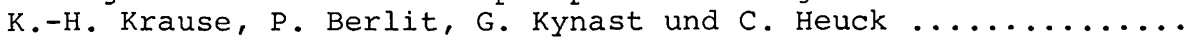

IgA-Mangel im Serum von Anfallskranken und ihren nahen Angehörigen

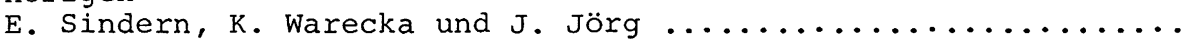

Complex Partial Seizures (CPS): Magnetic Resonance Imaging

(MRI) in Patients with Normal Cranial Computerized Tomography

(CCT) : Correlations with the EEG

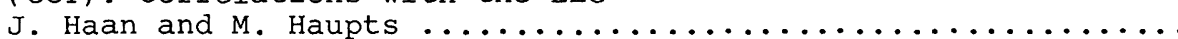

Intravenous Immunoglobulins in the Treatment of Intractable Epilepsy

K. Van Rijckevorsel-Harmant, 14. Delire, C. Sindic,

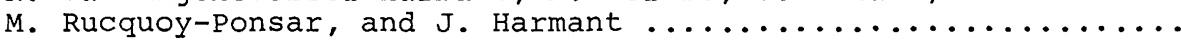

Therapeutic Response to Phenytoin in a Patient Suffering from Kleine-Levin Syndrome

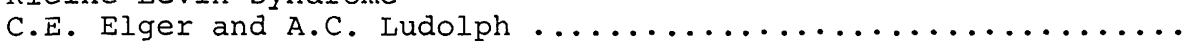

Postpartaler Krampfstatus bei 2 Schwestern - ein Reyeähnliches Krankheitsbild

D. Englert, E. Rösler, G. Reimann, F.J. von Baumgarten

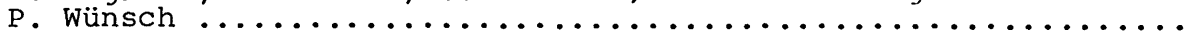


Klinische Bedeutung der postiktalen Serum-Prolaktinerhöhung

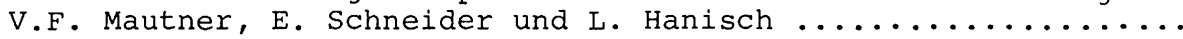

Mikroabsencen

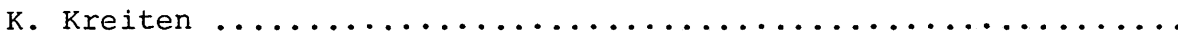

Carbamazepin (Tegretal) und Carbamazepin 400 ret. (Tegretal 400 ret.) Serumspiegel-Tagesprofile bei Anfallskranken

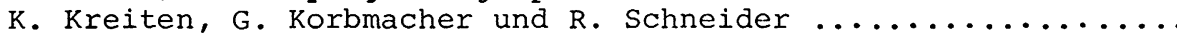

Epidemiology of Epilepsy in a Dutch Outpatient University Clinic

A. Keyser, H. Rwiza, M. Pool, H. Verweij, and Y. Hekster .....

Postersitzung - Spinale Krankheiten SEP

Topographie somatosensibler (SSEP) und visuell evozierter Potentiale (VEP) bei Morbus Huntington

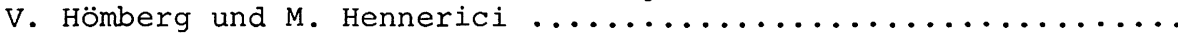

SEP-Verlaufsuntersuchungen bei Ischämie des Kaninchenrückenmarks

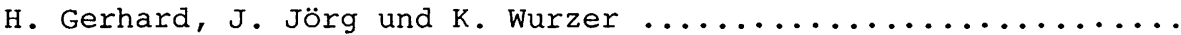

Subkortikale Komponenten der somato-sensibel evozierten potentiale (SEP): Befunde im Hirntod und bei Hirnstammläsionen

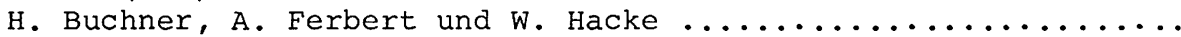

Serial SEP's and Intraoperative SEP Recording in a Patient with Syringomyelia. A Case Report

J.W. Vredeveld, P. Wuisman, J.J. Korten, and G. Spincemaille..

Langzeitergebnisse nach Zweit- und Mehrfachoperationen an lumbalen Bandscheibenvorfällen

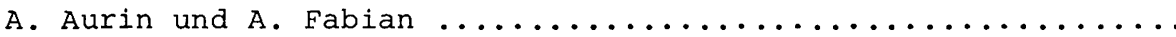

Zur Therapie der Strahlenmyelopathie

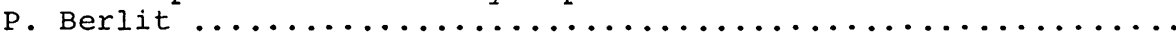

The Brown-Séquard Syndrome: True or False?

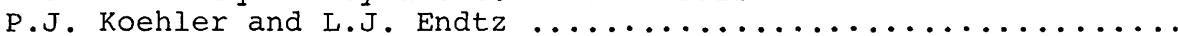

Quantification of the Effects of Antispastic Dirugs by Means of Flexor-Reflex Electromyography

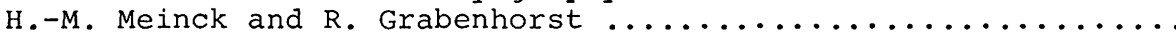

Spinal Extradural Lipomas: A Report on Three Patients R.A. Dierckx, A. Pallua, U. Mayr, F. Aichner, and

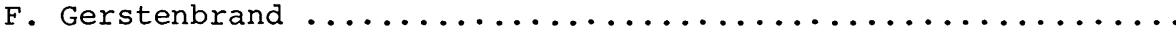

Postersitzung - Onkologie, metabolische Krankheiten

Electrophysiological Examinations in the Bielschowsky-Jansky Type of Neuronal Ceroid Lipofuscinosis

W. Tackmann, P. Vogel, and D. Yuhlendahl ................

Klinische Pharmakologie von natürlichem $\beta$-Interferon

U. Bogdahn, B. Fleischer, J. Hilfenhaus, H.J. Röthig,

P. Krauseneck, H. Przuntek, K.W. Pflughaupt und H.G. Mertens .. 
Zur Pharmakokinetik der Interferontherapie maligner Gliome

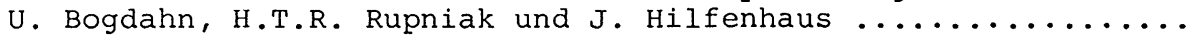

Lokale IgG-Produktion bei Meningeosis carcinomatosa

H.I. Schipper, A. Bardosi, C. Jacobi und K. Felgenhauer .......

Deutsch-österreichische Multi-Center-Studie zu malignen Gliomen

P. Krauseneck, H.G. Mertens, D. Messerer, P. Kleihues,

M. Bamberg, W. Dittmann, L. Gerhard, K. Heuser, U. Hobert,

H.B. Makoski, R. Poburski, G. Ransmayr, E. Richter und D. Volc

666

Die "Erlanger Malerstudie" (EMS) - keine Enzephalopathie bei

deutschen Baumalern

G. Triebig, K.-F. Druschky, S. Lehrl, W. Weidenhammer,

D. Claus, U. Weitbrecht, J. Reichwein, C. Lang, D. Penner,

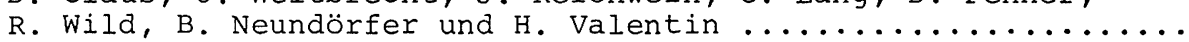

B-Vitaminmangel bei chronischem Alkoholismus: Bedeutung in

der Pathogenese metalkoholischer Erkrankungen

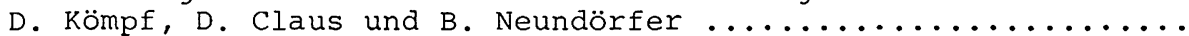

Riskfactors of "Normal" Alcohol Consumption, Oral Antidiabetic Drugs, and Treatment of Stroke

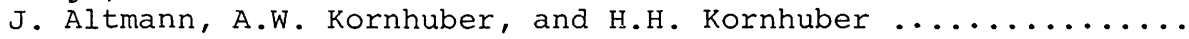

On the Road to Stroke: Hepatic Steatosis and Hyperinsulinaemia

Associated with Normal Alcohol Use in Young Males

B. Scheben, C. Henkler, A. Kornhuber, H.H. Kornhuber, V. Meier,

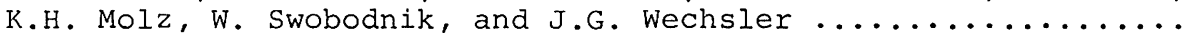

Zu Indikation und zeitpunkt der Strahlentherapie bei niedriggradigen Gliomen (WHO Grad I und II)

P. Krauseneck, E. Richter, W. Dittmann, H.-A. Müller, B. Ködel

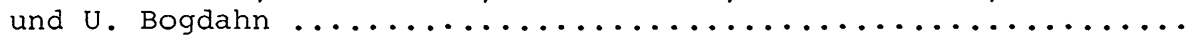

Postersitzung - Extrapyramidales System

Der Effekt von Budipin auf das MPTP-induzierte ParkinsonSyndrom bei Marmoset: Untersuchung der 5-HIAA-, HVA- und MHPG-Konzentrationen im Liquor

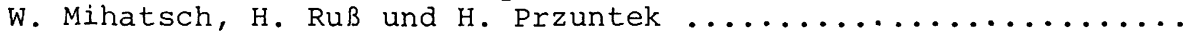

Standardisierte Verlaufsbeobachtungen des Therapieeffekts von Antiparkinson-Substanzen mit der motorischen Leistungsserie nach Schoppe

P.H. Kraus, W. Kuhn, P. Klotz, A. Fischer, F. von Baumgarten

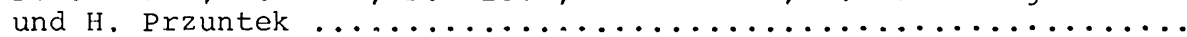

Der Orbicularis-oculi-Reflex bei einer Patientin mit MeigeSyndrom - vor und während erfolgreicher Behandlung mit Baclofen M. Schlenker, $H$. Buchner und $W$. Hacke $\ldots \ldots \ldots \ldots \ldots \ldots \ldots$

Torticollis spasmodicus - Verlauf, Therapie und Prognose

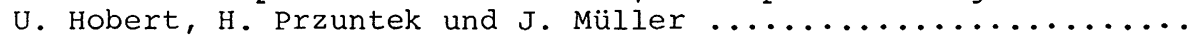

Parkinson-Therapie mit L-Dopa: Untersuchungen zur Pharmakokinetik von L-Dopa-Retardformulierungen

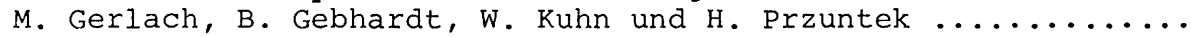

Subkutane Langzeitapplikationen von Lisurid bei schwerem Parkinson-Syndrom mit therapieresistenten Fluktuationen

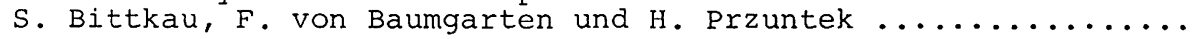


Vergleich elektromyographischer Befunde mit klinischen Schweregradskalen beim Parkinson-Syndrom

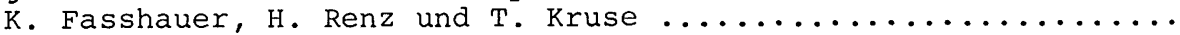

Morbus Parkinson und L-Dopa-Langzeitbehandlung: Sind Drug Holidays noch $\mathrm{zu}$ rechtfertigen?

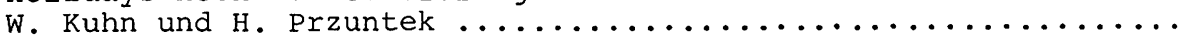

Quantifizierung von Antispastika-Akuteffekten durch Reflexelektromyographie

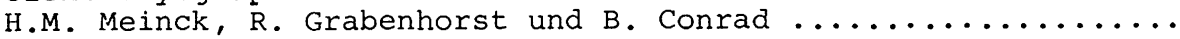

Postersitzung - Extrapyramidales System, Neuropsychologie

Klinik, Zusatzbefunde und Verlauf bei sporadischen

"kryptogenetischen" Kleinhirnatrophien

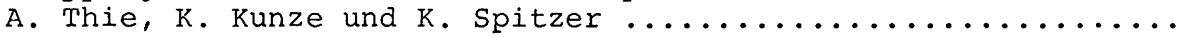

Ereigniskorrelierte Potentiale und Signaldetektion

T.F. Münte, H.J. Heinze, M. Scholz und H. Künkel ...........

EEG-Veränderungen unter Biperiden

C. Haag, B. Grözinger, W. Scherb, K.-P. Westphal, V. Diekmann

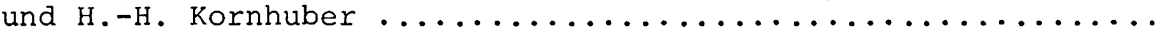

Erworbene Hirnleistungsstörungen und neurologische Residuen nach spontanen intrazerebralen Hämatomen

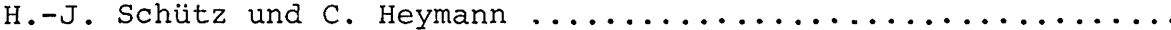

Der Benton-Test: ein einfaches Instrument zur Erfassung neuropsychologischer Ausfälle bei multipler Sklerose

U. Mann, L. Kappos, A. von der Wense und I. Haubitz .........

Höhere Packungsdichte informationsverarbeitender Fähigkeiten

in der linken Großhirnhemisphäre des Menschen

D. Bechinger, H.H. Kornhuber, H. Jung und E. Sauer ...........

Relationship Between Electroencephalographic (EEG) Synchronization and Plasma Dopamine-beta-hydroxylase (DBH) Activity in Patients

C.J.F. Kemperman, S.L.H. Notermans, and R. Wevers ..........

Motorische Einheiten im Frühstadium der Chorea Huntington

$M$. Schubert, R. Dengler, W. Wolf und A. Struppler ..........

Depression bei Morbus Parkinson

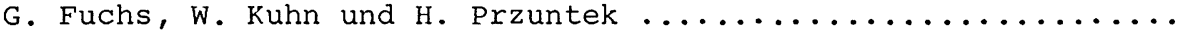

Postersitzung - Neuropsychologie

Sprachliche Automatismen in einem Sprachproduktionsmodell

G. Blanken, J. Dittmann, H. Johannsen-Horbach und

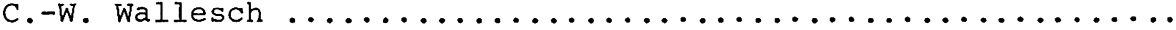

Kognitive Störungen des Handelns im Raum bei patienten mit Kleinhirnläsionen

A. Horn, J.C. Haas und $C .-W$. Wallesch $\ldots \ldots \ldots \ldots \ldots \ldots$

Neuropsychological Profile of Subtle Cognitive Dysfunctions in Excessive Alcohol Abusers

E. Thiery, M. Porto Carrero, and H. van der Eecken ........... 
Electrophysiological Evidence for Left Frontal Lobe Dominance in a Verbal Cognitive Learning Task

M. Lang, W. Lang, F. Uhl, and A. Kornhuber ..............

Picture Aphasiology

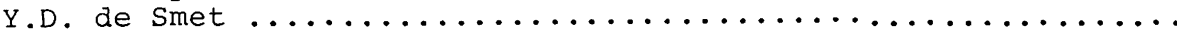

Untersuchungen zur sogenannten Hirnleistungsschwäche

J. Hunger, B. Leplow, C. Mohs, J. Kleim und U. Lamparter .....

Anarthrias with or Without Buccofacial Apraxias: Lesions'

Topography

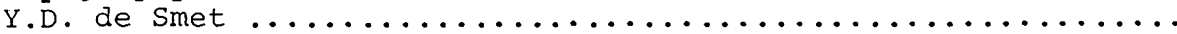

Gedächtnisuntersuchung zur Frage der Beeinträchtigung der

Hirnleistungsfähigkeit bei der Myasthenia gravis

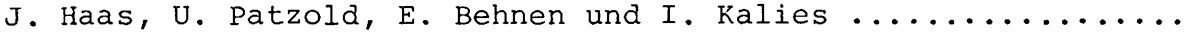

Spezifität einfacher apparativer psychomotorischer Untersuchungen bei der Quantifizierung des Parkinson-Syndroms

P. Klotz, A. Fischer, P.H. Kraus, B. Keck und H. Przuntek ....

Postersitzung - Verschiedenes

Kein Schutz gegen zervikale Plexus- und Wurzelzerreißung durch gebräuchliche sturzhelme

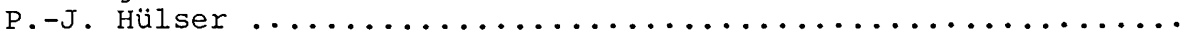

Epidurales Hämatom im Bereich der Lendenwirbelsäule unter Lowdose-Heparinbehandlung einer dialysepflichtigen Patientin

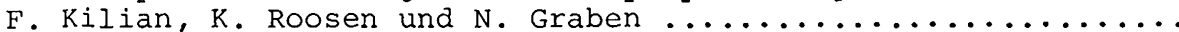

Krankenhausinformationssystem an der Neurologischen

Universitätsklinik Hamburg

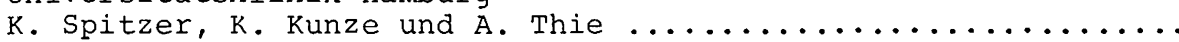

Realisierung eines Datenbankkonzeptes in der klinischen

Neurologie

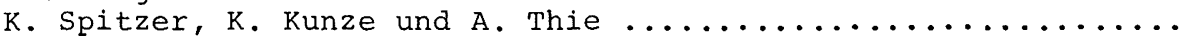

An Improved Home-Use Monitor to Detect Apnea Associated with Sudden Infant Death

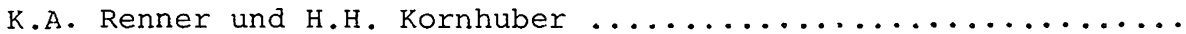

Der Erfekt von Budipin auf den Bing-Horton-Kopfschmerz

G. Reimann, W. Kohlhepp, D. Krüger und H. Przuntek .........

The Influence of Scopolamine on the Parameters of Vestibular Nystagmus

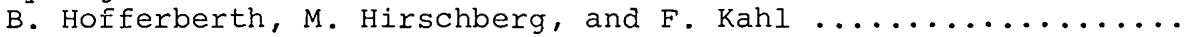

Undulating Peripheral Edema with Neurological Syndrome in

Two Children

C.J.F. Kemperman, F.J.M. Gabreëls, and W.O. Renier .........

A Possible Role of Nerve Growth Factor in Alzheimer's Disease

H. Gnahn, M. Näher, U. Besinger, and A. Struppler .......... 


\title{
Immunglobuline unter antiepileptischer Langzeitmedikation
}

\author{
K.-H. Krause, P. Berlit, G. Kynast und C. Heuck
}

Patienten und Methodik

Bei 492 Anfallskranken (288 Männer, 204 Frauen) im Alter von 20-40 Jahren unter antiepileptischer Langzeitmedikation wurden im Plasma mittels immunnephelometrischer Endpunktbestimmung (Lasernephelometer, Behringwerke, Marburg) die Immunglobuline $A, G$ und $M$ bestimmt und mit denen von 287 gleichaltrigen Normalpersonen verglichen (Wilcoxon-Test). Die Korrelationen mit durchschnittlicher Tagesdosis und Gesamtmenge an Antiepileptika sowie Therapiedauer wurden errechnet, die Werte bei den unter Monotherapie mit Phenytoin $(n=45)$, Primidon $(n=72)$, Carbamazepin $(n=50)$ und Valproat $(n=29)$ stehenden Patienten verglichen (außerdem mit den Medikamentenspiegeln korreliert), ebenso wie die Immunglobulinkonzentrationen bei idiopathischer und symptomatischer Epilepsie sowie bei bis zu 2 Jahren und über 10 Jahren behandelten Epileptikern. Möglichen Beziehungen zu Gingivahyperplasie und Leukozyten- sowie Lymphozytenzahl wurde nachgegangen.

\section{Ergebnisse}

Signifikante Abweichungen wiesen lediglich die männlichen Epileptiker beim IgG (Erhöhung) und IgM (Erniedrigung) auf (s. Tabelle 1). Korrelationen mit der Medikamentenmenge und Therapiedauer zeigten nur die weiblichen Anfallskranken (positiv, für IgA, IgG und IgM). Bei den Frauen lagen sämtliche Immunglobuline, bei den Männern nur IgA und IgG bei kurzer Behandlungsdauer niedriger als bei langer ( $s$. Tabelle 1). Die Immunglobulinspiegel der Patienten mit idiopathischer Epilepsie unterschieden sich nicht signifikant von denen mit symptomatischer. IgA lag bei den monotherapierten Männern für Phenytoin und Carbamazepin niedriger als für Primidon und Valproat. Die IgG-Spiegel waren bei den mit Valproat therapierten höher als bei den übrigen Epileptikern (s. Tabelle 1). Sichere Korrelationen mit Medikamentenspiegeln fanden sich nicht. Frauen mit Gingivahyperplasie hatten ein höheres IgA ( $p$ $<0,01)$ und IgM $(p<0,05)$ als solche ohne (s. Tabelle 1). Beziehungen zwischen den Immunglobulinen und der Lymphozyten- und Leukozytenzahl fanden sich nicht.

\section{Diskussion}

Die von mehreren Autoren gefundene IgA-Erniedrigung unter Phenytoin fand sich in unserer Studie bestätigt; für Carbamazepin sind die bisherigen Mitteilungen kontrovers, wir sahen hier ebenfalls niedrigere Werte. In der Mehrzahl wird in der Literatur die auch von uns gesehene Erniedrigung des IGM unter Antiepileptika beschrieben; unterschiedlich sind wiederum die Ergebnisse beim IgG, wo teils höhere - wie bei uns -, teil niedrigere Werte mitgeteilt wurden. Niedrigere IgA-Werte in der 
Tabelle 1. Immunglobulinspiegel im Plasma bei Epileptikern und Kontrollpersonen sowie in Abhängigkeit von Behandlungsdauex und Art der Medikation und von der Manifestation einer Gingivahyperplasie ( $M=$ männlich, $W=$ weiblich $)$

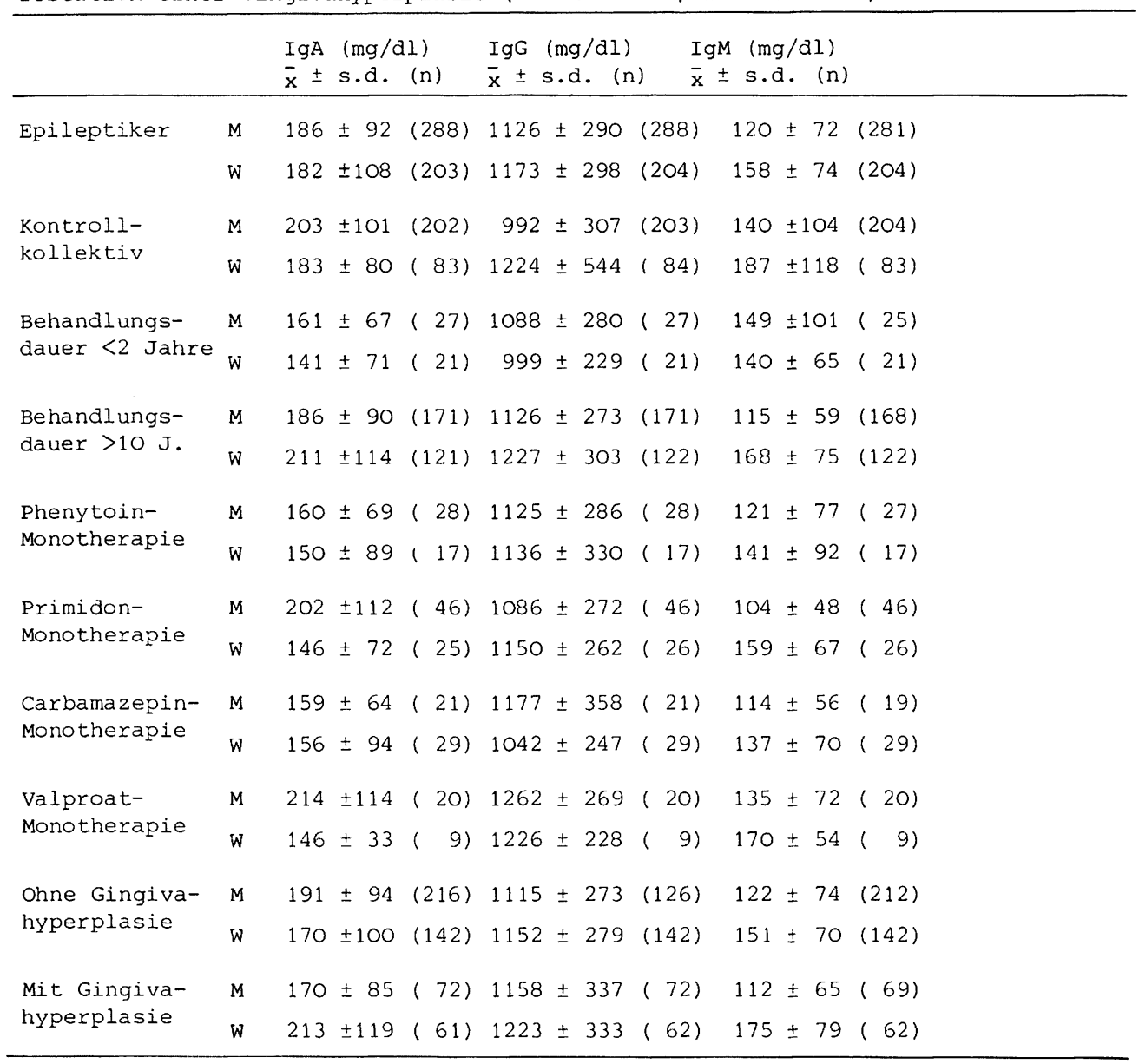

Anfangsphase der Therapie mit Anstieg im weiteren Verlauf wurden von Gilhus u. Aarli (1) beschrieben, wir sahen entsprechende veränderungen bei allen Immunglobulinen; die positiven Korrelationen zwischen IgG-Spiegeln und Medikamentendaten bei den Frauen können durch diesen Befund erklärt werden. Inwieweit der Immunstatus bei der Manifestation einer Gingivahyperplasie eine Rolle spielen könnte, bleibt unklar: die weiblichen Anfallskranken in unserer Studie mit Gingivahyperplasie hatten sogar höhere IgA- und IgM-Spiegel, bei den männlichen war die Tendenz gerade entgegengesetzt.

\section{Literatur}

1. Gilhus NE, Aarli JA (1981) The reversibility of phenytoin-induced IqA deficiency. J Neurol 226:53-61 